\title{
Étude de l'influence de la ramification du stolon sur la distribution des assimilats chez le trèfle blanc (Trifolium repens L.)
}

\author{
C. Robin, V. Guerin et A. Guckert \\ ENSAIA, laboratoire de phytotechnie INRA, 2, avenue de la forêt de Haye, 54500 Vandœuvre, France
}

(reçu le 12 décembre 1988; accepté le 17 juillet 1989)

Résumé - Les assimilats formés par les limbes et distribués vers les organes puits jouent un rôle capital dans la construction des organes végétatifs. De plus, l'allocation de carbone aux nodosités est garante d'un système fixateur efficient.

A l'aide d'un "phytomodèle" et de marquages courts au ${ }^{14} \mathrm{CO}_{2}$, nous avons déterminé le rôle de la ramification sur les flux de photoassimilats chez le trèfle blanc. Les données montrent clairement que l'interaction «âge de la ramification-position de la feuille source gère les relations entre les puits. La force du puits nodosités tend à chuter en présence d'une ramification surnuméraire en cours de développement. L'étude confirme le rôle tampon du stolon : l'activité de l'ensemble de l'organe de réserve est peu modifiée, quelles que soient les modalités du marquage. En revanche, la part allouée au stolon-fils est d'autant plus importante que la ramification est âgée, avec comme conséquence une moindre distribution au stolon parent. Un point végétatif à 4 feuilles reçoit encore du carbone issu des feuilles sources du stolon parent.

légumineuse - relations source-puits - compétition - stolon - assimilat - trèfle blanc

Summary - Effect of ramification of the stolon on the assimilates distribution in the white clover. Assimilates synthetized by the lamina and translocated to the sink organs play an important role in the building of the vegetative organs. Especially, the efficiency of nitrogen fixation depends on the carbon supply to the nodules.

With the help of a white clover "phytomodel" and short pulse labelling of single leaf with ${ }^{14} \mathrm{CO}_{2}$, we have determined the role of ramifications on assimilate flux to various plant organs. Two cases were distinguished (Figure 1):

- stolon without ramification, with only 8 leaves and 1 apex

- stolon with 1 ramification, 2 apices;

- ramification with 2 leaves $\left(R_{4}\right)$

- ramification with 4 leaves $\left(R_{8}\right)$ near the nodulated roots

Different leaves were chosen (Table I) in order to determine assimilate distribution :

- from the main stolon towards the ramification (fed leaves $F_{4}$ and $F_{8}$ );

- from the ramification towards the main stolon (fed leaves $F_{1}$ and $F_{2}$ : ramification $R_{4} ;$ fed leaves $F_{1}$ and $F_{4}$; ramification $R_{8}$ )

The ramifications $R_{4}$ and $R_{8}$ imported significant assimilates ${ }^{14} \mathrm{C}$ (Tables $1 / \mathrm{a} \& \mathrm{llb}$ ) from leaves of the main stolon. Consequently the sink strength of nodules decreased (Table III). ${ }^{14} \mathrm{C}$-photosynthate exportation from the ramifications towards the main stolon was high, with 38-52\% of the exported activity according to the labelled leaf (Table IVa). Position of the labelled leaf and of the branch stolon played a role in the assimilate distribution to the organs (Table $\mathrm{Nb}$ ) which influenced the sink strength of nodules and ramification. Branch stolon showed a high sink strength especially in the case of $R_{4}$ labelled leaf $f_{2}$ (Table V).

Whatever the labelled leaf position, this study confirms the buffering power of the whole stolon (main stolon + stolon of the ramification; Figure 2). On the other hand, the older the daughter branch, the higher the assimilate supply, and consequently the lower the allocation to the parent stolon.

legume - source-sink relationships - competition- stolon - assimilate - white clover

A.E. : Activité exportée

A.S.R. : Activité spécifique relative 


\section{INTRODUCTION}

Le trèfle blanc, dont le développement et la multiplication sont assujettis à la formation et la croissance d'un stolon, est une légumineuse fourragère très fréquente au sein des prairies permanentes.

Le stolon, organe à structure de tige, remplit de multiples fonctions : il assure la pérennité du trèfle en accumulant des réserves amylacées (Guckert et al., 1983); par ailleurs, en se ramifiant abondamment (croissance indéterminée), il conditionne le pouvoir de compétition de l'espèce et la colonisation du terrain.

L'apex du stolon assure l'élaboration de nouvelles feuilles. La rhizogénèse, ainsi que la formation ultérieure des nodosités sur les racines, ont lieu au niveau des entre-nœuds plus âgés. Les ramifications, très abondantes en phase active de croissance, sont initiées à l'aisselle des feuilles. Le rôle de puits des apex de stolons à l'égard des assimilats a été clairement établi (Harvey, 1970; Hoshino, 1972; Robin \& Guckert, 1987).

La distribution des photosynthétats aux nodosités des légumineuses pérennes en relation avec d'autres puits compétitifs a été peu étudiée. Elle varie avec le stade de développement de la plante et le rang de la feuille. (Crompton et al., 1981; Ismail \& Sagar, 1981).

Nous avons montré sur stolon "modèle" de trèfle blanc, sans ramifications, que l'alimentation des différents puits dépend de la position de la feuille donatrice (Robin \& Guckert, 1987; Robin et al., 1987). En revanche, peu de données sont disponibles quant au rôle des ramifications dans la répartition du carbone néo-fixé.

Le présent travail vise à étudier l'impact du puits supplémentaire représenté par le stolon-fils sur le schéma de répartition des photosynthétats précédemment établi. Le deuxième objectif de cette approche est de mettre en évidence les modalités d'allocation du carbone récent selon la position de ce nouveau point végétatif par rapport aux puits en place, pouvant induire notamment des relations de compétition vis-à-vis des autres apex et des nodosités.

\section{MATÉRIEL ET MÉTHODES}

\section{Matériel végétal}

Afin de réduire la variabilité génotypique importante chez le trèfle blanc (Ryle et al., 1981), les plantes sont obtenues par multiplication végétative d'apex de stolons issus d'un même pied-mère (type ladino, cv. regal), prélevé sur une parcelle du domaine expérimental de la Bouzule. Le choix d'un "phytomo- dèle" correspond au souci de réduire l'erreur expérimentale due en particulier aux variations de longueur entre plantes. Cette sélection vise à réduire les variations éventuelles de répartition induites par des différences de distance entre les sources et les puits (Cook et Evans, 1978; Cralle et al., 1987).

Les boutures sont cultivées sur un sable quartzeux en bac plastique (15 plantes par bac, correspondant à une densité de 90 plantes. $\mathrm{m}^{-2}$ ) en cellules phytotroniques, avec une photopériode de $16 \mathrm{~h}$, une thermopériode jour/nuit de $22 / 17^{\circ} \mathrm{C}$. Dix lampes $\mathrm{HQI} 400 \mathrm{~W}$ à vapeur de mercure délivrent un flux de photons de l'ordre de $290 \mu$ moles. $\mathrm{m}^{-2} . \mathrm{s}^{-1}$.

L'arrosage des bacs à partir d'une solution nutritive sans azote minéral (solution de Mac-Knight) est quotidien à raison de 100 à $150 \mathrm{ml}$. Les boutures sont inoculées en début de rhizogenèse avec une suspension de Rhizobium trifolii USDA 2063 cultivée en boîte de Petri.

\section{Conduite de la croissance}

La conduite de la croissance s'effectue en excisant les inflorescences et les ramifications «indésirables" à l'aisselle des feuilles du stolon principal. Ce développement dirigé permet l'induction de la croissance de ramifications (stolons secondaires), de position hiérarchisée sur le stolon principal. La Figure 1 schématise les différents types de conduite de la culture des boutures; 2 cas sont distingués :

- Le stolon sans ramification, ou plante «modèle», pourvu de 8 feuilles, avec un seul apex en croissance, pour lequel les contributions respectives des différentes feuilles source à l'alimentation carbonée ont été déterminées antérieurement (Robin et al., 1987)

- Le stolon avec ramification, se distinguant de la plante «modèle" par la présence d'un second point

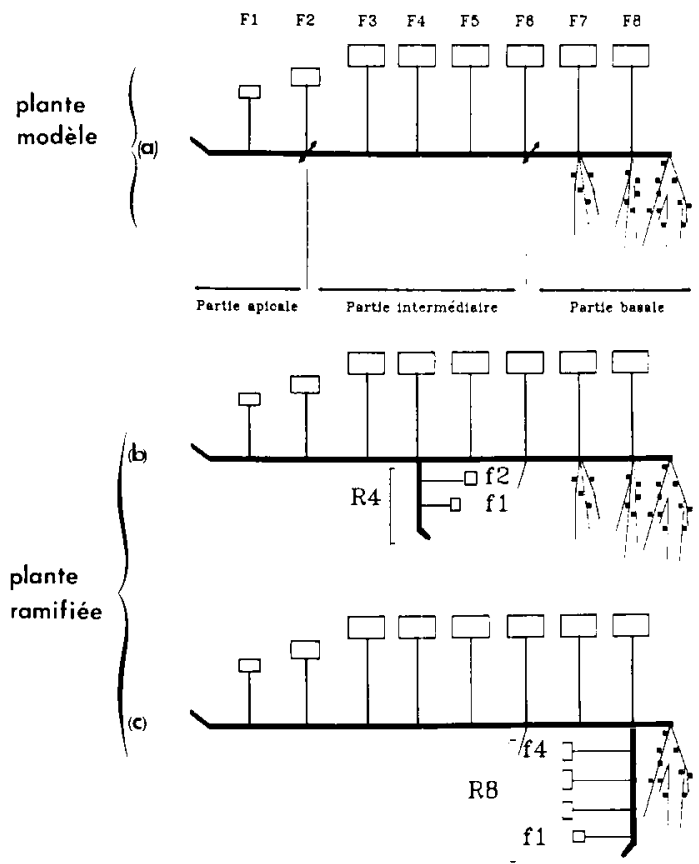

Fig. 1. Description des différents types de plantes engagées dans l'étude et plan d'échantillonnage de la plante en diffé rentes parties, en vue de la détermination de l'activité. (a): plante modèle (référence), (b) stolon ramifié en $F_{4}$, (c): stolon ramifié en $F_{8}$. 
végétatif en développement à l'aisselle d'une de ses 8 feuilles.

Deux types de ramifications ont été envisagés pour l'étude :

- Une ramification située à l'aisselle de la feuille $n^{\circ} 4$ (que nous distinguerons par $\mathrm{R}_{4}$ ), de position médiane sur le stolon principal, entre l'apex principal et le chevelu racinaire nodulé. $R_{4}$ n'a élaboré que 2 feuilles; cette ramification est la plus jeune initiée à partir du stolon principal.

- Une ramification à l'aisselle de la feuille $n^{\circ} 8\left(R_{8}\right)$, en position basale, à proximité des racines nodulées. $R_{8}$, constituée par 3 entre-nœuds et 4 feuilles dépliées, est déjà une ramification âgée.

\section{Transport et distribution des assimilats}

Six à sept semaines après le bouturage, les stolons ont atteint le stade 8 feuilles dépliées. Le Tableau 1 récapitule les modalités du marquage des différentes feuilles :

- sur le stolon principal d'une part (analyse des flux vers la ramification : feuille marquée $F_{4}$ et $F_{8}$ )

- sur le stolon secondaire d'autre part (analyse des flux de la ramification vers le stolon principal). Le marquage au ${ }^{14} \mathrm{C}$ sur la ramification concernera la plus jeune feuille dépliée et la plus âgée $\left(f_{1}\right.$ et $f_{2}$ pour $R_{4}, f_{1}$ et $f_{4}$ pour $R_{8}$ )

La plante «modèle» sera prise comme référence, avec les feuilles marquées $F_{4}$ et $F_{8}$ sur le stolon principal.

Le marquage a lieu $7 \mathrm{~h}$ après le début de la photopériode, selon le protocole décrit par Messier \& Guckert (1978). Chaque feuille est marquée individuellement à l'aide d'une chambre d'assimilation de faible volume $(50 \times 50 \times 2 \mathrm{~mm})$ dans laquelle le limbe est inséré hermétiquement sans lésion du pétiole. Le gaz marqué contenant $270 \mathrm{vpm}$ de $\mathrm{CO}_{2}\left(\mathrm{AS}=148.10^{7} \mathrm{~Bq} \cdot \mathrm{g}^{-1}\right.$ de carbone) est réparti uniformément dans la chambre à raison d'un débit de $120 \mathrm{ml}$. $\mathrm{min}^{-1}$.

Les paramètres du marquage ont été fixés précédemment (Robin \& Guckert, 1987). Le temps de charge est de 2 min et le temps de transit (chasse) de $6 \mathrm{~h}$ (déterminé par une cinétique de distribution) permet la migration des assimilats ${ }^{14} \mathrm{C}$ dans l'ensemble de la plante. Ce temps de chasse est adopté par d'autres auteurs (Scheidegger, 1983)

\section{Echantillonnage et traitement des tissus végétaux marqués}

A l'issue des $6 \mathrm{~h}$ de chasse, les plantes marquées sont échantillonnées en 3 parties (Fig. 1):

- partie apicale (PA), incluant les feuilles $F_{1}$ et $F_{2}$ - partie intermédiaire $(P I): F_{3}$ à $F_{6}$, avec ou sans ramification $R_{4}$.

- partie basale $(P B): F_{7}$ à $F_{8}$, avec ou sans ramification $\mathrm{R}_{8}$.

Chacune de ces parties est séparée en ses organes constitutifs (feuilles, stolon, racines, nodosités), qui sont congelés rapidement à l'aide de Carboglace.

Après lyophilisation et broyage fin, les échantillons sont brûlés dans un four "Oxymat Intertechnique» IN 4101. La radioactivité est ensuite déterminée en scintillation liquide (mélange scintillant Combutron, compteur Tri-carb Packard).

\section{Paramètres de l'analyse}

Les quantités de carbone assimilé sont fonction de la surface foliaire et de l'âge des tissus chlorophylliens. Les feuilles concernées par le marquage présentent des caractéristiques physiologiques différentes selon leur position sur le stolon (Robin et al., 1987).

A partir des résultats bruts de comptage en scintillation, les paramètres suivants permettent d'analyser et de comparer la répartition des photosynthétats issus des feuilles marquées dans les différentes parties et les différents organes de la plante modèle.

\section{L'activité en \% de l'activité exportée (\%AE)}

Elle exprime la part des assimilats exportés par le limbe source vers un organe donné:

$\% A E=A$ (d'un organe ou d'un ensemble d'organes) $\times 100$

A (plante - limbe marqué)

$A=$ Activite $(B q)$

\section{L'activité spécifique relative (ASR)}

Ce paramètre sans unité est le seul utilisable à des fins de comparaison des caractéristiques de distribu-

Tableau 1. Etude des flux vers la ramification et vers le stolon principal : position des feuilles engagées dans les marquages sur le stolon principal $(F)$ et sur les ramifications (f)

Position des feuilles marquées

Plante modèle

Ramif. $R_{4}$

Ramif. $R_{8}$

Marquage sur stolon

primaire
$F_{4}$

$\mathrm{F}_{8}$
$\mathrm{F}_{4}$

$F_{8}$

Marquage sur stolon

secondaire $f_{1} \quad f_{1}$ 
tion des assimilats à partir de feuilles d'âges physiologiques différents (Mor \& Halevy, 1980; Cralle \& Heichel, 1985; Robin et al., 1987). L'activité spécifique relative (ASR) prend uniquement en compte le critère "transport" en annulant les différences d'efficience des limbes vis-à-vis de l'assimilation du ${ }^{14} \mathrm{CO}_{2}$.

ASR $=B q \cdot g^{-1} \mathrm{MS}$ (d'un organe ou d'un ensemble d'organes)

Bq.g-1 MS (plante - limbe marqué)

\section{Traitement statistique des données}

Les résultats ont fait l'objet d'une analyse de variance avec classement des moyennes selon le test de Newman-Keuls. Les valeurs moyennes (issues de 4 répétitions) suivies de la même lettre ne sont pas significativement différentes au seuil de $5 \%$.

\section{RÉSULTATS}

Chez le trèfle blanc, la position de la feuille source joue un rôle dans la distribution des assimilats (Robin et al., 1987). L'apex du stolon se montre un organe fortement compétiteur du reste de la plante et des nodosités, en particulier pour les photosynthétats alloués par les jeunes feuilles. Les nodosités sont alimentées en carbone essentiellement par les feuilles ágées $\left(F_{4}\right.$ et suivantes). Ces résultats obtenus sur stolon «modèle" seront pris comme données de référence afin :

- de dégager le rôle compétiteur de la ramification en croissance sur la dynamique des photoassimilats à partir des feuilles source;

- de déterminer l'importance des flux vers la ramification d'une part, de la ramification vers le stolon principal d'autre part.

\section{Etude des flux du stolon primaire vers le sto- Ion secondaire}

La distribution des photoassimilats marqués sera d'abord exprimée par l'activité exportée puis par l'activité spécifique relative au niveau de chaque partie de la plante et de chaque organe d'autre part.

\section{Répartition de l'actlvité (en \% de l'activité exportée).}

Distribution entre les 3 parties de la plante selon la position de la ramification (Tableau lla)

Cas de la ramification $R_{4}$, feuille marquée $F_{4}$ Les résultats montrent que le schéma global de répartition des flux vers les différentes parties de la plante, établi sur plante modèle, est peu modifié. La ramification $R_{4}$ importe néanmoins $12,9 \%$ de l'activité aux dépens du reste de la partie intermédiaire.

\section{Cas de la ramification $R_{8}$, feuille marquée $F_{8}$}

Les différences sont plus importantes : les exportations vers $R_{8}$ représentent près de $29 \%$ de l'activité. La partie intermédiaire est moins marquée $(17,7$ contre $24,1 \%$ pour la partie intermédiaire de la plante de référence) au profit de la partie basale ( $44 \%$ contre $28 \%$ pour la partie basale de la plante de référence).

Distribution entre organes selon la position de la ramification (Tableau Ilb)

Cas de la ramification $R_{4}$, feuille marquée $F_{4}$ Nous n'observons pas de différences notables entre plante modèle et plante ramifiée en ce qui concerne les pourcentages d'activité des organes de l'ensemble de la plante (feuilles, stolon et système racinaire). II en résulte que les importations aux organes foliaires (6\%) et au stolon de la ramification $(6 \%)$ se font aux dépens des organes de la partie parente.

Cas de la ramification $R_{8}$, feuille marquée $F_{8}$ En présence de la ramification $R_{8}$, les feuilles présentent une activité supérieure consécutive à une importation de $13 \%$ d'activité aux feuilles de la partie ramifiée. L'activité du stolon reste stable, avec près de $11 \%$ alloués au stolon de la ramification. Les importations vers les nodosités sont fortement diminuées $(3,5 \%$ contre $8,2 \%$ pour les nodosités de la plante de référence).

\section{Répartition de l'activité exprimée en activité spécifique relative (Tableau III)}

\section{ASR du stolon}

L'ASR du stolon chute fortement en présence d'une ramification. Le stolon principal (partie parente) et le stolon secondaire (ramification) présentent des ASR nettement différentes : quelle que soit la position de la ramification, le stolon secondaire montre une ASR bien supérieure à celle du stolon principal, supérieure également à l'ASR du stolon de la plante "modèle".

\section{ASR des nodosités}

Chez la plante modèle (marquage $\mathrm{F}_{4} \mathrm{OU} \mathrm{F}_{8}$ ), l'ASR des nodosités est plus élevée que celle du stolon.

En présence d'une ramification, I'ASR des nodosités chute nettement, avec un effet particulièrement évident dans le cas de la ramification $R_{8}$ (ASR inférieure à 0,2 ). 
Tableau lla. Etude des flux en provenance d'une feuille du stolon principal: activité (en \% de l'activité exportée) des différentes parties de la plante et de la ramification. Les valeurs entre parenthèses représentent l'activité de la ramification uniquement.

Les valeurs moyennes suivies de la même lettre ne sont pas significativement différentes au risque 5\% (test de Newman-Keuls).

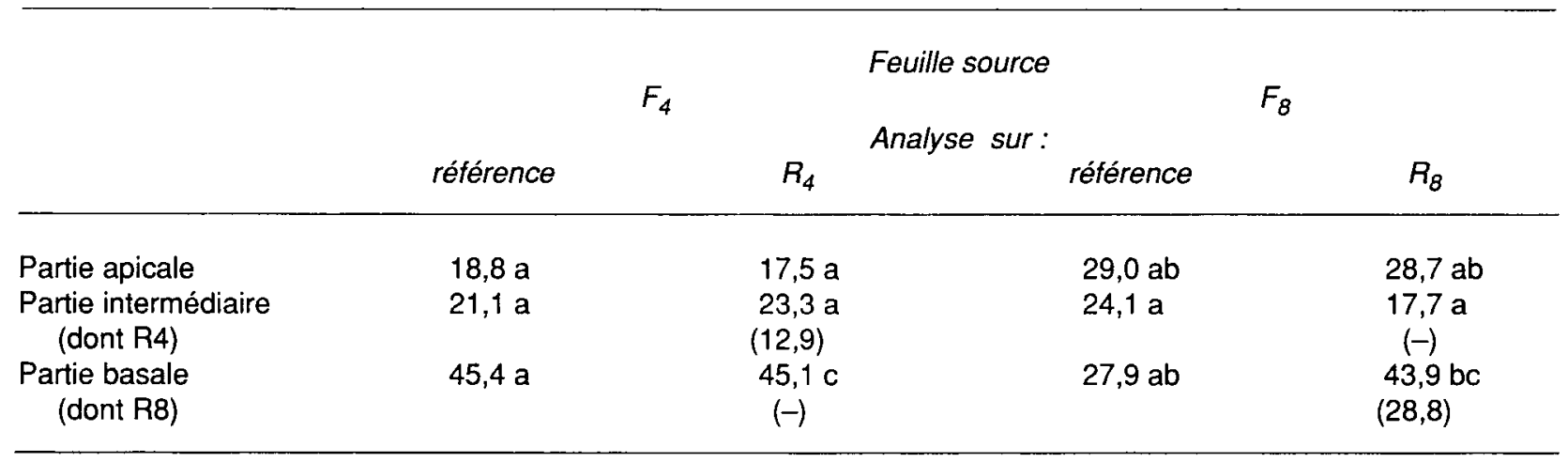

Tableau Ilb. Etude des flux en provenance d'une feuille du stolon principal: activité (en \% de l'activité exportée) vers les organes de l'ensemble de la plante (total feuilles, total stolon, racines, nodosités), vers les organes du stolon principal uniquement (feuilles I et stolon I) et de la ramification uniquement (feuilles II et stolon II).

Les valeurs moyennes suivies de la même lettre ne sont pas significativement différentes au risque $5 \%$ (test de Newman-Keuls).

\begin{tabular}{lcccc}
\hline & & \multicolumn{2}{c}{ Feuille source } \\
& Stol. réf. & $F_{4}$ & Analyse sur : & \multicolumn{2}{c}{$F_{8}$} \\
& & $R_{4}$ & Stol. réf. & $R_{8}$ \\
\hline Feuilles I & 20,1 & 15,6 & 32,1 & 30,9 \\
Feuilles II & - & 6,0 & - & 13,0 \\
Total feuilles & $20,1 \mathrm{bc}$ & $21,6 \mathrm{bc}$ & $32,1 \mathrm{~cd}$ & $43,9 \mathrm{~d}$ \\
Stolon primaire & 32,4 & 25,7 & 29,7 & 21,2 \\
Stolon secondaire & - & 6,0 & - & 10,9 \\
Total stolon & $32,4 \mathrm{~cd}$ & $31,7 \mathrm{~cd}$ & $29,7 \mathrm{~cd}$ & $31,1 \mathrm{~cd}$ \\
Racines & $23,2 \mathrm{bc}$ & $20,6 \mathrm{bc}$ & $9,9 \mathrm{ab}$ & $11,7 \mathrm{ab}$ \\
Nodosités & $9,6 \mathrm{ab}$ & $12,2 \mathrm{ab}$ & $8,2 \mathrm{ab}$ & $3,5 \mathrm{a}$ \\
\hline
\end{tabular}

Tableau III. Etude des flux en provenance d'une feuille du stolon principal : activité spécifique relative de l'ensemble du stolon et des nodosités, du stolon primaire uniquement.

Les valeurs moyennes suivies de la même lettre ne sont pas significativement différentes au risque $5 \%$.

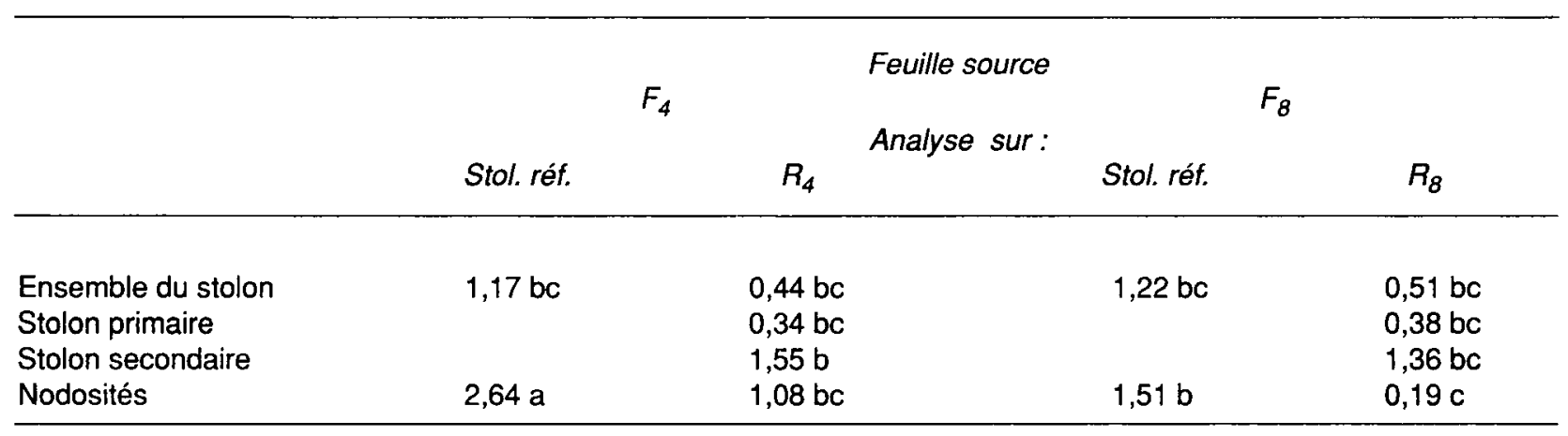




\section{Etude des flux du stolon secondaire vers le stolon primaire.}

La feuille la plus jeune et la feuille la plus âgée de chaque ramification ont été utilisées tour à tour comme source de photoassimilats- ${ }^{14} \mathrm{C}$.

\section{Répartition de l'activité en \% de l'activité exportée.}

Distribution entre les 3 parties de la plante (Tableau IVa) selon la position de la feuille source et de la ramification

Cas de la ramification $R_{4}$, feuilles marquées $f_{1}$ et $f_{2}$

Les activités des parties apicale et basale sont fortement diminuées par rapport à la plante de référence, surtout dans le cas où les assimilats sont fournis par la plus jeune feuille de la ramification. La partie intermédiaire présente une forte activité (supérieure à $40 \%$ ) due à la présence de la ramification ( $26 \%$ d'AE).

Les flux vers le stolon principal sont importants $\left(41 \%\right.$ pour la feuille $f_{1}, 52 \%$ pour la feuille $f_{2}$ la plus proche du stolon principal)

Cas de la ramification $R_{8}$, feuilles marquées $f_{1}$ et $\mathrm{f}_{4}$

La partie basale bénéficie de la présence de la ramification $(66 \%$ d'AE, dont la moitié pour la ramification à elle seule). II en résulte que les flux vers les parties intermédiaires et surtout apicales sont fortement réduits par rapport au phytomodèle, surtout pour la feuille source $f_{1}$.

Les flux vers le stolon parent s'élèvent à $43 \%$ et $38 \%$ pour la feuille $f_{1}$ et la feuille $f_{4}$, respectivement.

Tableau IVa. Etude des flux en provenance d'une feuille de la ramification: activité (en \% de l'activité exportée) des différentes parties de la plante, de la ramification (les valeurs entre parenthèses représentent l'activité de l'ensemble de la ramification) et du stolon principal.

Les valeurs moyennes d'une même ligne suivies de la même lettre ne sont pas significativement différentes au risque $5 \%$ (test de Newman-Keuls).

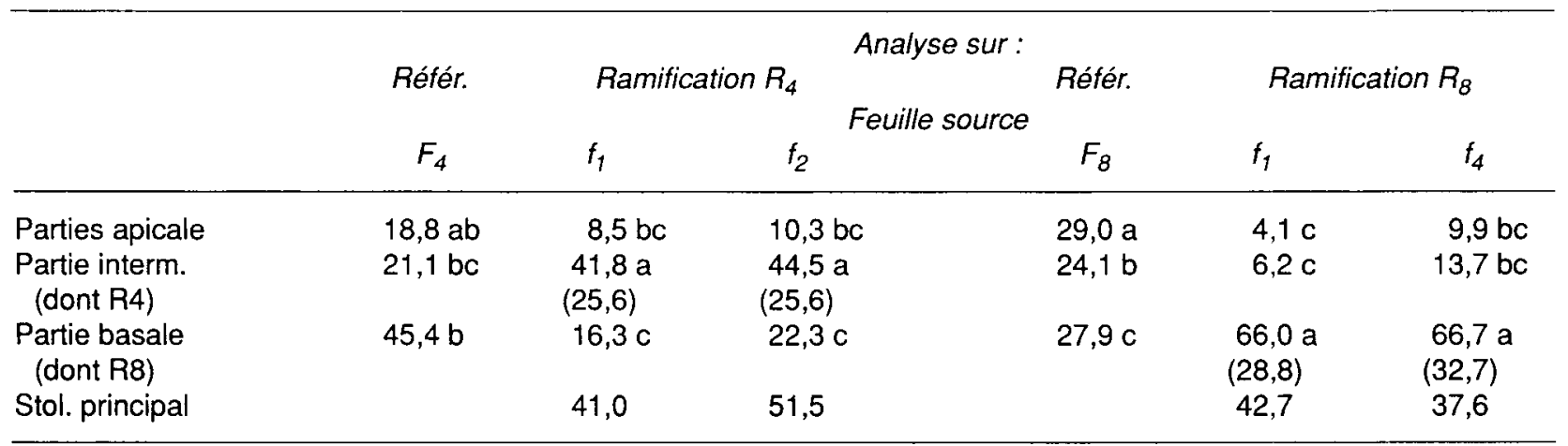

Tableau IVb. Etude des flux en provenance d'une feuille de la ramification: activité (en \% de l'activité exportée) des organes de l'ensemble de la plante (total feuilles, total stolon, racines, nodosités), des organes du stolon principal uniquement (feuilles I et stolon I) et de la ramification uniquement (feuilles II et stolon II).

\begin{tabular}{lccrcrcr} 
& Référ. & \multicolumn{2}{c}{ Ramification $R_{4}$} & Analyse sur : & Référ. & \multicolumn{2}{c}{ Ramification $R_{8}$} \\
& $F_{4}$ & $f_{1}$ & $f_{2}$ & Feuille source & $F_{8}$ & $f_{1}$ & $f_{4}$ \\
\hline Feuilles I & 20,1 & 18,2 & 16,0 & 32,1 & 9,4 & 21,7 \\
Feuilles II & - & 2,4 & 8,9 & - & 5,6 & 12,4 \\
Total feuilles & 20,1 & 20,6 & 24,9 & 32,1 & 15,0 & 34,1 \\
Stolon primaire & 32,4 & 10,2 & 15,6 & 29,7 & 28,0 & 14,1 \\
Stolon secondaire & - & 25,8 & 16,7 & - & 5,3 & 20,3 \\
Total stolon & 32,4 & 36,0 & 32,3 & 29,7 & 33,3 & 34,4 \\
Racines & 23,2 & 7,6 & 16,3 & 9,9 & 22,9 & 17,4 \\
Nodosités & 9,6 & 2,6 & 3,7 & 8,2 & 4,6 & 4,0 \\
\hline
\end{tabular}


Distribution entre les organes (Tableau IVb) selon la position de la feuille source et de la ramification

Cas de la ramification $R_{4}$ feuilles marquées $f_{1}$ et $f_{2}$

L'activité du stolon reste stable quelle que soit la position de la feuille source. Les modifications de répartition sont nettes pour les racines nodulées; les exportations vers les nodosités, à partir de la feuille $\mathfrak{f}_{1}$ notamment, sont faibles $(2,6 \%$ contre près de $10 \%$ pour les nodosités de la plante modèle).

Cas de la ramification $R_{8}$, feuilles marquées $f_{1}$ et $f_{4}$.

La baisse d'activité des organes foliaires (en particulier ceux du stolon parent) par rapport à la plante de référence semble bénéficier aux racines, sans répercussions au niveau des nodosités (dont l'activité est réduite de moitié par rapport aux données du stolon modèle). On observe néanmoins que l'activité des nodosités est plus importante pour $R_{8}$ que pour $R_{4}$.

\section{Répartition de l'activité en activité spécifique relative (Tableau V)}

\section{ASR du stolon}

Quels que soient les cas, I'ASR du stolon est identique à l'ASR du stolon de la plante de référence. Le stolon secondaire montre par contre une ASR très élevée (supérieure à 5), dépassant de loin celle du stolon principal. L'ASR du stolon secondaire $R_{4}$ est nettement plus élevée que celle du stolon $R_{8}$.

\section{ASR des nodosités}

\section{Cas de $R_{4}$}

La force de puits des nodosités tend à diminuer, en particulier pour les assimilats issus de la feuille $f_{1}$ (ASR $=0,8$ contre 2,6 pour la plante modèle). Elle est inférieure à l'ASR du compartiment stolon.

\section{Cas de $R_{8}$}

L'ASR chute seulement pour la feuille $f_{1}$ (non significatif), feuille source la plus éloignée des racines nodulées. La force du puits nodosités est équivalente à celle du compartiment stolon.

\section{DISCUSSION}

Ce type de démarche visant à déterminer le rôle de puits des apex est plus couramment appréhendé par la technique de décapitation (Hoshino, 1972; Crafts-Brandner et al., 1983), de décortication annulaire ou "girdling" (Carlson et Brun, 1984), ou d'excision, ombrages et stress azoté, (Egli et al., 1985).

Les résultats mettent en évidence l'importance des interactions trophiques entre les stolons dans la stratégie de multiplication végétative du trèfle blanc. Une ramification est à la fois source et puits d'assimilats; chaque feuille du stolon secondaire concernée par notre étude est à la fois importatrice et exportatrice.

Le caractère hétérotrophe des ramifications est net. Les jeunes feuilles et l'apex du stolon sont des puits majeurs. Les points végétatifs présentent une proportion de tissus méristèmatiques et de cellules jeunes beaucoup plus importante que le stolon parent. La répartition des assimilats dépend de nombreux facteurs et en particulier de la taille des puits. Selon Harvey (1970), seul un stolon avec 5 feuilles développées cesse d'importer des assimilats. Scheidegger (1983) précise que plus de $50 \%$ des assimilats sont transférés vers l'apex. Warembourg et al. (1984) notent que ce sont les organes en forte croissance qui attirent le plus d'assimilats

Tableau V. Etude des flux en provenance d'une feuille de la ramification: activité spécifique relative du stolon et des nodosités, du stolon primaire uniquement, du stolon secondaire uniquement.

Les valeurs moyennes suivies de la même lettre ne sont pas significativement différentes au risque $5 \%$.

\begin{tabular}{|c|c|c|c|c|c|c|}
\hline & \multicolumn{6}{|c|}{ Analyse sur: } \\
\hline & Stolon réf. & \multicolumn{2}{|c|}{ Ramification $R_{4}$} & Stolon réf. & \multicolumn{2}{|c|}{ Ramification $R_{8}$} \\
\hline & $F_{4}$ & $f_{1}$ & $f_{2}$ & $F_{8}$ & $f_{1}$ & $f_{4}$ \\
\hline Total stolon & $1,17 \mathrm{~cd}$ & $1,40 \mathrm{~cd}$ & $1,48 \mathrm{~cd}$ & $1,22 \mathrm{~cd}$ & $1,22 \mathrm{~cd}$ & $1,60 \mathrm{~cd}$ \\
\hline $\begin{array}{l}\text { Stol. primaire } \\
\text { Stol secondaire }\end{array}$ & & $0,82 \mathrm{~d}$ & $0,74 \mathrm{~d}$ & & $0,38 d$ & $\begin{array}{l}0,93 \mathrm{~d} \\
5,33 \mathrm{~cd}\end{array}$ \\
\hline Nodosités & $2,64 \mathrm{~cd}$ & $0,76 \mathrm{~d}$ & $\begin{array}{r}4,00 \mathrm{~d} \\
1,12 \mathrm{~cd}\end{array}$ & $1,51 \mathrm{~cd}$ & $1,30 \mathrm{~cd}$ & $1,49 \mathrm{~cd}$ \\
\hline
\end{tabular}


récents chez le soja. Hoshino (1974) avait montré une forte dominance apicale; après excision de l'apex, le ${ }^{14} \mathrm{C}$ est distribué aux bourgeons latéraux à proximité de la feuille marquée. De plus, la dominance apicale tend à s'atténuer pour les zones plus éloignées de l'apex.

Les feuilles source de la ramification exportent une grosse partie des assimilats vers le stolon parent (plus de $40 \%$ ), avec un effet "position de la feuille" dans le cas de $R_{4}$ : l'allocation est plus importante pour la feuille la plus âgée de la ramification (52\%), la plus proche du stolon parent. La ramification $R_{4}$ présente une force de puits très importante et nettement supérieure à celle de $R_{8}$, constituant la ramification la plus âgée, dont les besoins en carbone sont assurés par un nombre plus élevé de feuilles adultes.

L'allocation de carbone aux nodosités est réduite en présence d'une ramification; une relation de compétition s'établit entre ces organes à fonctions physiologiques différentes, en faveur de la fonction de croissance et développement. Cette compétition est sous-tendue par les notions de taille de puits (Cook \& Evans, 1978) et de force de puits (fonction du produit de son activité par sa taille: Warren-Wilson, 1967, cité par Pinto, 1980). Selon Walker \& Ho (1977), la force de puits serait plus déterminée par son activité métabolique que par sa taille (distinguant les organes de réserve des organes à forte activité respiratoire). Ryle et al., (1981) remarquent que l'exportation aux ramifications est réduite en présence de nodosités. Laperriere (1984) a noté une augmentation de la fixation symbiotique après une excision de l'apex secondaire.

L'ensemble du stolon est composé de 2 sousensembles (stolon principal + stolon secondaire) à forces de puits très différentes. Nous avons schématisé les pourcentages d'activité exportée dans l'ensemble du stolon selon toutes les modalités de marquage (Fig. 2, schéma de synthèse);

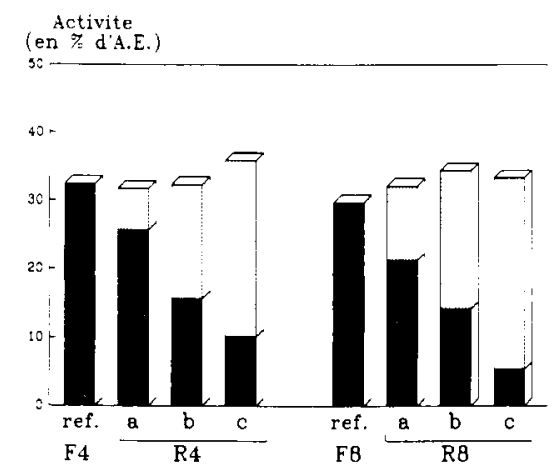

Fig. 2. Activité (en \% de l'activité exportée) du stolon sans ramification (référence $F_{4}$ ou $F_{8}$ ) ou avec ramification: stolon

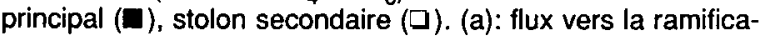
tion (b) et (c): flux de la ramification vers le stolon principal (b: feuille marquée la plus âgée, c: feuille marquée 1). quelle que soit la position de la ramification et de la feuille source, les pourcentages d'activité sont remarquablement stables (entre 30 et $36 \%$ d'AE), confirmant ainsi le rôle "tampon" de cet organe (Robin et Guckert, 1987). La distribution des photoassimilats se partage entre le stolon principal et le stolon secondaire; elle semble régie par la position de la feuille marquée et la position de la ramification.

L'influence de l'apex secondaire est plus nette pour les feuilles source du point végétatif; la fonction de puits du stolon est d'autant plus focalisée vers le stolon secondaire que la feuille donatrice est jeune.

\section{CONCLUSIONS}

Le développement du trèfle blanc est donc largement contrôlé par l'allocation d'assimilats carbonés des feuilles sources (organes photosynthétiques) aux organes puits consommateurs (méristèmes apicaux et nodosités). Nos résultats mettent en évidence des relations de compétition pour les photoassimilats entre les différents apex d'une part et entre les apex et les nodosités d'autre part.

Il semble donc que le stade de développement de la plante, conditionnant la présence de points végétatifs plus ou moins nombreux, exerce, avec les paramètres de l'environnement, une influence déterminante sur la distribution du carbone.

L'étape ultérieure de cette étude visera à étudier l'allocation de carbone à l'organe reproducteur, puis à modéliser ces flux qui régissent les mouvements de carbone dans la plante.

\section{RÉFÉRENCES}

Carlson D.R. \& Brun W.A. (1984) Alteration of ${ }^{14} \mathrm{C}$ assimilate partitioning in leaves of soybeans having reproductive loads at one node. Plant Physiol. 75, 887890

Cook M.G. \& Evans L.T. (1978) Effect of relative size and distance of competing sinks on the distribution of photosynthetic assimilates in wheat. Aust. J. Plant Physiol. 5, 495-509

Crafts-Brandner S.J., Below F.E., Harper J.E. \& Hageman R.H. (1983) Metabolism of carbon and nitrogen of soybean seedlings in response to vegetative apex removal. Plant Physiol. 73, 6-10

Cralle H.T. \& Heichel G.H. (1985) Interorgan photosynthate partitioning in Alfalfa. Plant Physiol. 79, 381-385

Cralle H.T., Heichel G.H. \& Barnes D.K. (1987) Photosynthate partitioning in plants of alfalfa population selected for high and low nodule mass. Crop Sci. 27, 96-100

Crompton H.J., Lloyd-Jones C.P. \& Hill-Cottingham D.G. (1981) Translocation of labelled assimilates follo- 
wing photosynthesis of ${ }^{14} \mathrm{CO}_{2}$ by the field bean, Vicia faba. Physiol. Plant. 51, 189-194

Egli D.B., Guffy R.D., Meckel L.W. \& Legget J.E. (1985) The effect of source-sink alterations on soybean seed growth. Ann. Bot. 55, 395-402

Guckert A., Damay J., Treillet L., Balandreau J., Bardin R. \& Chalamet A. (1983) Etude au champ de la fixation d'azote par le trèfle blanc (Trifolium repens L.). Fourrages $94,61-86$

Harvey H.J. (1970) Patterns of assimilate translocation in Trifolium repens. In: W.C. research, Oc. Symp. $n^{\circ} 6$, Brit. Grassl. Soc. 181-186

Hoshino M. (1972) Translocation and accumulation of assimilates in forage plants. Jap. Agric. Res. Quart., 6 (3)

Hoshino M. (1974) Translocation and accumulation of assimilates and phosphorus in Ladino clover. Bull. Natl. Grassl. Res. Inst. 5, 35-84

Ismail A.M.A. \& Sagar S.G. (1981) The influence of leaf age, leaf position and sinks on the rate of export and partition of ${ }^{14} \mathrm{C}$ at different stages of development following assimilation of ${ }^{14} \mathrm{CO}_{2}$ by a single leaf of Vicia faba L.. J. Hort. Sci. 56 (1), 55-63

Laperriere C. (1984) Etude de la fixation d'azote par le trèfle blanc (Trifolium repens L.). Aspects biologiques et agronomiques. Thèse $3^{\circ}$ Cycle, ENSAIA Nancy, $99 \mathrm{pp}$

Messier J. \& Guckert A. (1978) Contribution à la mesure de la photosynthèse au champ par la méthode utilisant le $\mathrm{CO}_{2}$ marqué. Bull. Ec. Natl. Sup. Agron. Nancy, XX, 31-45
Mor Y. \& Halevy A.H. (1980) Promotion of sink activity of developing rose shoots by light. Plant Physiol. 66, 990-995

Pinto C.M. (1980) Régulation de la photosynthèse par la demande d'assimilats: mécanismes possibles. Photosynthetica 14, 611-634.

Robin C. \& Guckert A. (1987) Etude de la répartition des assimilats chez le trèfle blanc (Trifolium repens L.). Les colloques de l'INRA $n^{\circ} 37,175-180$

Robin C., Chone T. \& Guckert A. (1987) Rôle de la position de la feuille dans l'assimilation et le transport du carbone chez le trèfle blanc (Trifolium repens L.). Agronomie 7 (8), 599-605

Ryle G.J.A., Powell C.E. \& Gordon A.J. (1981) Patterns of ${ }^{14} \mathrm{C}$-labelled assimilate partitioning in red and white clover during vegetative growth. Ann. Bot. 47, 505-514

Scheidegger U.C. (1983) Bildung und Verwendung der Photosyntheseproduckte bei Oekotypen von Weissklee (Trifolium repens L.) Diss. ETH n $n^{\circ} 7226$, Zürich, $64 \mathrm{pp}$

Walker A.J. \& Ho L.C. (1977) Carbon translocation in tomato: carbon import and fruit growth. Ann. Bot. 41, 813-823

Warembourg F.R., Haegel B., Fernandez M. \& Montange D. (1984) Distribution et utilisation des assimilats carbonés en relation avec la fixation symbiotique d'azote chez le soja (Glycine max L. Merrill). Plant Soil 82, 163-178 